\title{
Dynamical features of reaction-diffusion fronts in fractals
}

\author{
Vicenç Méndez, ${ }^{1}$ Daniel Campos, ${ }^{2, *}$ and Joaquim Fort ${ }^{3, \dagger}$ \\ ${ }^{1}$ Departamento de Medicina, Universitat Internacional de Catalunya, c./Gomera s/n, 08190-Sant Cugat del Vallés, Barcelona, Spain \\ ${ }^{2}$ Grup de Física Estadística, Departamento de Física, Universitat Autònoma de Barcelona, E-08193 Bellaterrra, Spain \\ ${ }^{3}$ Departamento de Física, Universitat de Girona, Campus de Montilivi, 17071 Girona, Catalonia, Spain
}

(Received 12 September 2003; published 29 January 2004)

\begin{abstract}
The speed of front propagation in fractals is studied by using (i) the reduction of the reaction-transport equation into a Hamilton-Jacobi equation and (ii) the local-equilibrium approach. Different equations proposed for describing transport in fractal media, together with logistic reaction kinetics, are considered. Finally, we analyze the main features of wave fronts resulting from this dynamic process, i.e., why they are accelerated and what is the exact form of this acceleration.
\end{abstract}

DOI: 10.1103/PhysRevE.69.016613

PACS number(s): 05.45.Df, 05.40.-a, 87.10.+e

\section{INTRODUCTION}

Reaction-diffusion models are based in general on the partial differential equation

$$
\frac{\partial P(x, t)}{\partial t}=D \frac{\partial^{2} P(x, t)}{\partial x^{2}}+f(P)
$$

where $P(x, t)$ is the density of particles at time $t$ at the position $x, D$ is the diffusion coefficient, and $f(P)$ is the growth (reaction) function. The characteristic wave front solutions that arise from these models make them suitable for many different applications which cover biological [1] and human [2] invasions, forest fires [3], epidemics [4], tumor growth [5], etc.

In spite of this, reaction-diffusion equations are often criticized, by arguing that such simple models cannot account for the complexity of real systems. Specifically, how the intricate features of spatial systems must be modeled is still a current problem. Some efforts have been made in the last years both to show the need for models able to predict spatial complexity [6] and propose some possible solutions [7]. Among these proposals, one of the most attractive and recurrent ones consists of assuming that the spatial structures exhibit self-similarity properties at a certain range of scales, so fractal scaling formalism may be considered.

In this work, we try to show some of the main concepts involved in adapting reaction-diffusion to self-similar spatial systems. It is well known that the mean-square displacement of a random walker in a fractal object fulfills the subdiffusive behavior [8]

$$
\left\langle x^{2}\right\rangle \sim t^{2 / d_{w}},
$$

where $d_{w} \geqslant 2$ is the random-walk dimension of the fractal (for $d_{w}=2$ one recovers the classical case). The main advances in the field of transport in fractals have been focused on finding a transport equation for the probability density $P(x, t)$ of finding a random walker at time $t$ at a distance $x$

\footnotetext{
*Email address: daniel.campos@uab.es

${ }^{\dagger}$ Email address: joaquim.fort@udg.es
}

from its starting point. At present, it is known that for all particles starting from the same origin and for sufficiently large times and distances, the following form of the probability density $[9,10]$

$$
\begin{gathered}
P(x, t) \sim \exp \left[-c\left(\frac{x}{t^{1 / d_{w}}}\right)^{u}\right], \\
u=\frac{d_{w} d_{\min }}{d_{w}-d_{\min }}
\end{gathered}
$$

is valid for a large class of fractals. In Eq. (4), $d_{\min }$ is the fractal dimension of the minimum distance between points of the fractal [11]. The classical, Euclidean case corresponds to $d_{w}=2, d_{\min }=1$ and, therefore, $u=2$ so that the solution is a Gaussian.

Transport equations proposed to date have tried to reproduce results (2) - (4) somehow. The first attempt was made by O'Shaugnessy and Procaccia (SP) [12]. They derived from scaling and renormalization arguments the SP equation

$$
\frac{\partial P}{\partial t}=\frac{1}{x^{d_{f}-1}} \frac{\partial}{\partial x}\left[D(x) x^{d_{f}-1} \frac{\partial P}{\partial x}\right]
$$

where $d_{f}$ is the geometric fractal dimension of the fractal, $D(x)=D^{*} x^{2-d_{w}}$, and the constant $D^{*}$ is a kind of diffusion coefficient. The exact solution for this equation is known and its second moment behaves like $t^{2 / d_{w}}$, in agreement with Eq. (2). However, this solution lacks the scaling (3) and (4) predicted by numerical simulations.

Later on, Giona and Roman (GR) constructed the fractional GR equation [13]

$$
\frac{\partial^{1 / d_{w}} P}{\partial t^{1 / d_{w}}}=-A\left(\frac{\partial P}{\partial x}+\frac{\kappa}{x} P\right),
$$

where $\kappa=\left(d_{f}-1\right) / 2$. The exact solution of Eq. (6) is also known and yields again the behavior $t^{2 / d_{w}}$ for the second moment of $P$, Eq. (2). However, the exact solution for $P$ and Eq. (6) do not recover the Gaussian solution and the $d_{f}$-dimensional classical diffusion equation, respectively. 
To overcome these difficulties, more recently a generalization of the SP equation including a temporal fractional derivative

$$
\frac{\partial^{\gamma} P}{\partial t^{\gamma}}=\frac{1}{x^{d_{f}-1}} \frac{\partial}{\partial x}\left[D(x) x^{d_{f}-1} \frac{\partial P}{\partial x}\right]
$$

has been proposed [14].

Finally, very recently we have proposed a partial differential equation [the Campos-Méndez-Fort (CMF) equation] from a stronger physical justification than the previous models, which also improves the results obtained by previous approaches [9].

Our goal is to find the analytical relationship for the speed of fronts when the above transport equations couple to a reaction process modeled by logistic-KPP (KolmogorovPetrovskii-Piskunov) kinetics. We employ the method of reduction of the reaction-transport equation to a HamiltonJacobi. Also, we are able to derive in some cases the speed of the fronts by using the local-equilibrium (LE) approach [15] when the spatial correlations are small.

\section{SP EQUATION WITH REACTION}

First of all we consider that the reaction-diffusion process in a fractal is described by the equation for the probability density of O'Shaughnessy and Procaccia [Eq. (5)] coupled to a KPP kinetic term

$$
\frac{\partial P}{\partial t}=\frac{1}{x^{d_{f}-1}} \frac{\partial}{\partial x}\left(D^{*} x^{d_{f}-d_{w}+1} \frac{\partial P}{\partial x}\right)+r P(1-P) .
$$

\section{A. Reduction to a Hamilton-Jacobi equation}

In order to find the asymptotic speed for the traveling wave fronts in Eq. (8) we will first make use of HamiltonJacobi dynamics [16]. The starting point is the hyperbolic scaling procedure $t \rightarrow t / \varepsilon, x \rightarrow x / \varepsilon$, and the representation of the rescaled probability density function $P^{\varepsilon}(x, t)$ $=P(x / \varepsilon, t / \varepsilon)$ in WKB form

$$
P^{\varepsilon}(x, t)=\exp \left(-\frac{G^{\varepsilon}(x, t)}{\varepsilon}\right), \quad G^{\varepsilon}(x, t) \geqslant 0,
$$

where the action functional $G^{\varepsilon}$ has to be found. It follows from Eq. (9) that, as long as the function $G(x, t)$ $=\lim _{\varepsilon \rightarrow 0} G^{\varepsilon}(x, t)$ is positive, the rescaled field $P^{\varepsilon}(x, t) \rightarrow 0$ as $\varepsilon \rightarrow 0$. The boundary of the set where $G(x, t)>0$ can be regarded as a reaction front. Therefore, we may argue that the reaction front position $x(t)$ can be determined from the equation $G(x(t), t)=0$. Substituting Eq. (9) into the rescaled equation for $P^{\varepsilon}(x, t)$ we find the equation for $G^{\varepsilon}(x, t)$

$$
\begin{aligned}
-\frac{\partial G^{\varepsilon}}{\partial t}= & -\varepsilon^{d_{w}-1} D^{*}\left(d_{f}-d_{w}+1\right) x^{d_{w}+1} \frac{\partial G^{\varepsilon}}{\partial x} \\
& +\varepsilon^{d_{w}-2} D^{*} x^{2-d_{w}}\left(\frac{\partial G^{\varepsilon}}{\partial x}\right)^{2} \\
& -\varepsilon^{d_{w}-1} D^{*} x^{2-d_{w}} \frac{\partial^{2} G^{\varepsilon}}{\partial x^{2}}+r .
\end{aligned}
$$

The first and third terms in the right-hand side of Eq. (10) have the same order of magnitude and in the asymptotic limit $(\varepsilon \rightarrow 0)$ both terms may be neglected in front of the second term, and in consequence the Hamilton-Jacobi for the front propagation in a fractal media is

$$
\frac{\partial G}{\partial t}+\left(\frac{\varepsilon}{x}\right)^{d_{w}-2} D^{*}\left(\frac{\partial G}{\partial x}\right)^{2}+r=0
$$

where $G(x, t)=\lim _{\varepsilon \rightarrow 0} G^{\varepsilon}(x, t)$. In order to find the solution for $G(x, t)$ in Eq. (11) we make use of the Hamilton equations

$$
\begin{gathered}
\frac{d x(\tau)}{d \tau}=\frac{\partial H}{\partial p(\tau)}=2\left[\frac{\varepsilon}{x(\tau)}\right]^{d_{w}-2} D^{*} p(\tau), \\
\frac{d p(\tau)}{d \tau}=-\frac{\partial H}{\partial x(\tau)}=\left(d_{w}-2\right) \frac{\varepsilon^{d_{w}-2}}{x(\tau)^{d_{w}-1}} D^{*} p(\tau)^{2},
\end{gathered}
$$

where $H \equiv-\partial G / \partial t$ and $p(\tau) \equiv \partial G / \partial x(\tau)$ and $\tau$ stands for the temporal coordinate. The solution of the Hamilton-Jacobi equation (29) can be written as

$$
G(x, t)=\min _{x(\cdot)}\left\{\int_{0}^{t} L(x, \tau) d \tau: x(0)=0, x(t)=x\right\},
$$

where $L(x, \tau)=p(\tau)[d x(\tau) / d \tau]-H$ is the Lagrangian associated with $H$. Integrating Eq. (12), one has $x(\tau)$ $=x(\tau / t)^{2 / d_{w}}$ under the boundary conditions $x(0)=0, x(t)$ $=x$, and

$$
L(x, \tau)=\frac{x^{d_{w}}}{t^{2} D^{*} \varepsilon^{d_{w}-2} d_{w}^{2}}-r
$$

and from Eq. (13)

$$
G(x, t)=\frac{x^{d_{w}}}{t D^{*} \varepsilon^{d_{w}-2} d_{w}^{2}}-r t .
$$

The speed of the front is computed from $G(x, t)=0$, and after inverting the hyperbolic scaling one finally has the exact expression for the speed of the front

$$
v(t)=2\left[\frac{D^{*} r}{d_{w}^{d_{w}-2} t^{d_{w}-2}}\right]^{1 / d_{w}} \sim t^{\left(2 / d_{w}\right)-1},
$$

which describes a decelerated front, and we note that the acceleration depends on the parameter $d_{w}$. 


\section{B. Local-equilibrium approach}

The local-equilibrium hypothesis has been very successful in thermodynamics (where one assumes a Gibbs equation with the local values of temperature, pressure, etc.), radiative transfer, astrophysics (where one assumes a radiative emission-absorption equilibrium at the local temperature), etc. Here, we will apply such a heuristic approximation to the problem of front propagation. Equation (8) can be rewritten as

$$
\begin{aligned}
\frac{\partial P}{\partial t}= & \frac{1}{x^{d_{w}-1}} D^{*}\left(d_{f}-d_{w}+1\right) \frac{\partial P}{\partial x}+\frac{1}{x^{d_{w}-2}} D^{*} \frac{\partial^{2} P}{\partial x^{2}} \\
& +r P(1-P) .
\end{aligned}
$$

This equation has the form

$$
\frac{\partial P}{\partial t}=D(x) \frac{\partial^{2} P}{\partial x^{2}}-V(x) \frac{\partial P}{\partial x}+r P(1-P),
$$

which is an advection-reaction-diffusion equation in a medium moving at speed $V(x)$. For homogeneous values of $D$ and $V$, the speed of fronts is obviously

$$
v=2 \sqrt{r D}+V
$$

which is nothing but Fisher's speed $2 \sqrt{r D}$ (which holds for an observer attached to the moving medium), as seen by an observer moving with speed $-V$. This result $v=2 \sqrt{r D}+V$ may be also derived by using the linearization and variational techniques in Ref. [17]. We propose that, if inhomogeneities are sufficiently smooth, this equation should hold locally, so that we obtain the local-equilibrium prediction

$$
v_{L E} \simeq 2 \sqrt{r D(x)}+V(x)
$$

which for Eq. (15) yields

$$
v_{L E}=\frac{d x}{d t} \simeq 2 \frac{\sqrt{r D^{*}}}{x^{\left(d_{w}-2\right) / 2}}-\frac{D^{*}\left(d_{f}-d_{w}+1\right)}{x^{d_{w}-1}} .
$$

Note that if one takes the hyperbolic scaling $x \rightarrow x / \varepsilon, t$ $\rightarrow t / \varepsilon$ with $\varepsilon \rightarrow 0$ in Eq. (19), one observes that the second term on the right-hand side (rhs) of (19) is negligible, compared to the first one, and Eq. (19) can be written as

$$
\frac{d x}{d t} \simeq 2 \frac{\sqrt{r D^{*}}}{x^{\left(d_{w}-2\right) / 2}} \quad \text { for } \quad x, t \rightarrow \infty
$$

which may be integrated to yield $x(t)=\left[d_{w} \sqrt{r D^{*}} t\right]^{2 / d_{w}}$, where we have assumed the initial condition $x(0)=0$. Finally, the speed may be obtained as

$$
v_{L E}=\frac{d x}{d t} \simeq 2\left[\frac{D^{*} r}{\left(d_{w} t\right)^{d_{w}-2}}\right]^{1 / d_{w}} \sim t^{\left(2 / d_{w}\right)-1} \text { for } t \rightarrow \infty,
$$

which coincides with Eq. (14). The asymptotic speed does not depend on the fractal dimension $d_{f}$, which could be

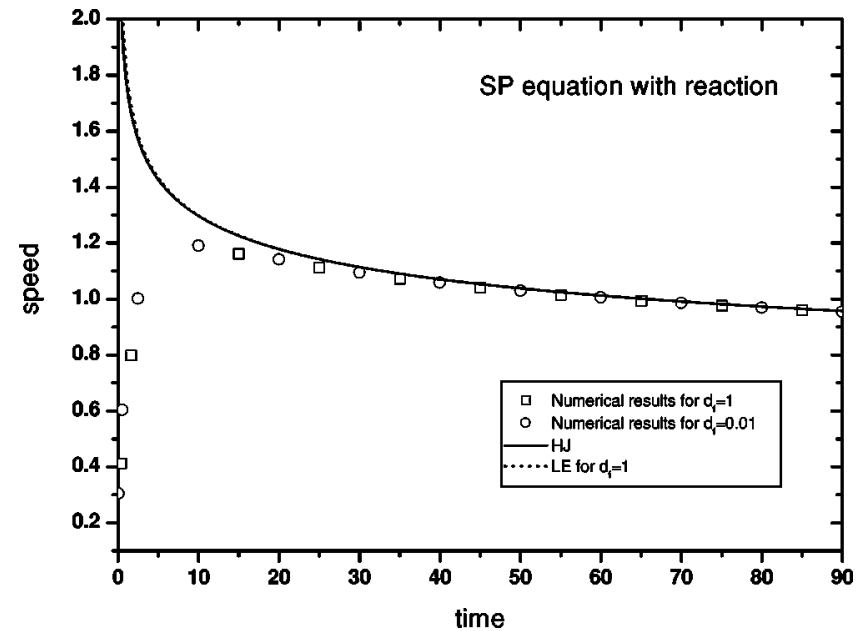

FIG. 1. Comparison between numerical results for the speed of fronts of Eq. (8) for $d_{f}=1$ and for $d_{f}=0.01$ and the analytical results from the HJ method given in Eq. (14) (solid line) and the numerical result for the LE method obtained from Eq. (21) with $d_{f}=1$. All magnitudes are dimensionless. We observe a very good agrement between analytical and numerical solutions in the asymptotic regime. It is clear that $d_{f}$ does not affect the value of the speed for large times. We have taken $D^{*}=r=1$ and $d_{w}=2.32$.

something surprising at first sight. In order to further explore this point, we have solved numerically Eq. (8), under the initial condition $P(x, 0)=1$ for $x<0$ and $P(x, 0)=0$ for $x$ $>0$, for two very different values of $d_{f}$ and we have observed, as we show in Fig. 1, that the speed depends weakly on $d_{f}$ only at the very initial transient and loses this dependence as time grows. Moreover, in Fig. 1 we compare the speed given in Eq. (14) or (20) with the speed obtained from Eq. (19). To do this we must first solve Eq. (19). Under $x(0)=0$ we obtain

$$
t=\frac{y}{d_{w} \sqrt{r D^{*}}}+\frac{\left(d_{f}-d_{w}+1\right)}{2 d_{w} r} \ln \left(1-\frac{2 y \sqrt{r / D^{*}}}{d_{f}-d_{w}+1}\right),
$$

where $x=y^{2 / d_{w}}$. In Fig. 1 we have solved numerically the transcendent equation (21) and evaluated $d x / d t$ to obtain the speed. Note that the effect of $d_{f}$ [which appears in Eq. (21) but not in Eq. (20)] on the asymptotic speed of the front is inappreciable. This shows that it is justified to neglect the second term in the rhs of Eq. (19), as done above.

\section{GR EQUATION WITH REACTION}

In this section we study the speed of fronts for the fractional advection equation GR (6) with reaction

$$
\frac{\partial^{1 / d_{w} P}}{\partial t^{1 / d_{w}}}=-A\left(\frac{\partial P}{\partial x}+\frac{\kappa}{x} P\right)+r P(1-P),
$$




$$
\begin{aligned}
\frac{\partial^{1 / d_{w} P}}{\partial t^{1 / d_{w}}} & =\frac{1}{\Gamma\left(1-\frac{1}{d_{w}}\right)} \frac{\partial}{\partial t} \int_{0}^{t} \frac{P\left(x, t^{\prime}\right)}{\left(t-t^{\prime}\right)^{1 / d_{w}}} d t^{\prime} \\
& =\frac{1}{\Gamma\left(1-\frac{1}{d_{w}}\right)} \frac{\partial}{\partial t} \int_{0}^{t} z^{-1 / d_{w}} P(x, t-z) d z
\end{aligned}
$$

and $z=t-t^{\prime}$. As in the preceding section we first take the asymptotic limit for large space and time by employing the hyperbolic scaling. The left-hand side of Eq. (6) is

$$
\begin{aligned}
\frac{\partial^{1 / d_{w} P^{\varepsilon}}}{\partial t^{1 / d_{w}}} & =\frac{\varepsilon}{\Gamma\left(1-\frac{1}{d_{w}}\right)} \int_{0}^{t / \varepsilon} z^{-1 / d_{w}} \frac{\partial}{\partial t} P^{\varepsilon}(x, t-\varepsilon z) d z \\
& =\frac{\varepsilon}{\Gamma\left(1-\frac{1}{d_{w}}\right)} \int_{0}^{t / \varepsilon} z^{-1 / d_{w}}\left(\frac{\partial P^{\varepsilon}}{\partial t}-\frac{\partial^{2} P^{\varepsilon}}{\partial t^{2}} \varepsilon z+\cdots\right),
\end{aligned}
$$

where $P^{\varepsilon}(x, 0)=0$ for $x>0$, and making use of Eq. (9) one finds

$$
\frac{\partial^{1 / d_{w} P^{\varepsilon}}}{\partial t^{1 / d_{w}}}=\frac{-\left(\partial_{t} G^{\varepsilon}\right) e^{-G^{\varepsilon} / \varepsilon}}{\Gamma\left(1-\frac{1}{d_{w}}\right)} \int_{0}^{t / \varepsilon} z^{-1 / d_{w}} e^{z \partial_{t} G^{\varepsilon}} d z+O(\varepsilon) .
$$

The right-hand side of Eq. (6) is transformed into

$$
\begin{array}{r}
-A\left(\varepsilon \frac{\partial P^{\varepsilon}}{\partial x}+\varepsilon \frac{\kappa}{x} P^{\varepsilon}\right)+r P^{\varepsilon}\left(1-P^{\varepsilon}\right) \\
=A \frac{\partial G^{\varepsilon}}{\partial x} e^{-G^{\varepsilon} / \varepsilon}+r e^{-G^{\varepsilon} / \varepsilon}+O(\varepsilon) .
\end{array}
$$

Taking $\varepsilon \rightarrow 0$ one gets the Hamilton-Jacobi equation

$$
\left(-\frac{\partial G}{\partial t}\right)^{1 / d_{w}}=A \frac{\partial G}{\partial x}+r
$$

From the Hamilton equations one has the Lagrangian

$$
L(x, t)=\frac{x}{A t d_{w}}\left[\left(d_{w}-1\right)\left(\frac{x}{A t d_{w}}\right)^{1 /\left(d_{w}-1\right)}-d_{w} r\right],
$$

and the speed of the front will be

$$
v=A d_{w}\left(\frac{r d_{w}}{d_{w}-1}\right)^{d_{w}-1}
$$

which is time independent.

\section{FRACTIONAL SP EQUATION WITH REACTION}

Another equation proposed is the fractional version of the $\mathrm{SP}$ equation (7) which with reaction takes the form

$$
\frac{\partial^{\gamma} P}{\partial t^{\gamma}}=\frac{1}{x^{d_{f}-1}} \frac{\partial}{\partial x}\left[D^{*} x^{d_{f}-d_{w}+1} \frac{\partial P}{\partial x}\right]+r P(1-P),
$$

where the fractional time derivative is defined as [14]

$$
\frac{\partial^{\gamma} P}{\partial t^{\gamma}}=\frac{1}{\Gamma(1-\gamma)} \frac{\partial}{\partial t} \int_{0}^{t} \frac{P\left(x, t^{\prime}\right)}{\left(t-t^{\prime}\right)^{\gamma}} d t^{\prime}
$$

Taking the hyperbolic scaling and Eq. (9) in the limit $\varepsilon \rightarrow 0$ one finds the Hamilton-Jacobi equation

$$
\left(-\frac{\partial G}{\partial t}\right)^{\gamma}=D^{*} x^{2-d_{w}} \varepsilon^{d_{w}-2}\left(\frac{\partial G}{\partial x}\right)^{2}+r
$$

From the Hamilton equations one obtains $p(\tau)$ $=B x(\tau)^{-1+d_{w} / 2}$ and

$$
\frac{x(\tau)^{d_{w} / 2}}{d_{w} / 2}=\frac{2 D^{*} B}{\gamma} \varepsilon^{d_{w}-2}\left(r+D^{*} \varepsilon^{d_{w}-2} B^{2}\right)^{(1 / \gamma)-1} \tau,
$$

where $B$ is an integration constant to be determined from the condition $x(\tau=t)=x$, and therefore

$$
\frac{x^{d_{w} / 2}}{d_{w} / 2}=\frac{2 D^{*} B}{\gamma} \varepsilon^{d_{w}-2}\left(r+D^{*} \varepsilon^{d_{w}-2} B^{2}\right)^{(1 / \gamma)-1} t .
$$

On the other hand, the Lagrangian function is

$$
L(x, t)=\left(r+D^{*} \varepsilon^{d_{w}-2} B^{2}\right)^{-1+1 / \gamma}\left[\left(\frac{2}{\gamma}-1\right) D^{*} \varepsilon^{d_{w}-2} B^{2}-r\right]
$$

From $G(x, t)=0$ we have

$$
D^{*} \varepsilon^{d_{w}-2} B^{2}=\frac{r \gamma}{2-\gamma}
$$

and inserting this into Eq. (25) one has

$$
x^{d_{w} / 2}=d_{w} \sqrt{D^{*} / \gamma} \varepsilon^{\left(d_{w}-2\right) / 2}\left(\frac{r}{2-\gamma}\right)^{(2-\gamma) / 2 \gamma} 2^{(1-\gamma) / \gamma} t
$$

and taking $d x / d t$ the speed is

$$
v(t)=2\left[\frac{2^{2(1-\gamma) / \gamma}}{\gamma}\left(\frac{r}{2-\gamma}\right)^{(2-\gamma) / \gamma} \frac{D^{*}}{\left(d_{w} t\right)^{d_{w}-2}}\right]^{1 / d_{w}} \sim t^{\left(2 / d_{w}\right)-1}
$$

once the hyperbolic scaling is inverted. Note that for $\gamma=1$, Eq. (14) is recovered. However, the scaling law for the speed of the front in time is the same as for $\gamma=1$ in Eq. (20) so that the fractional derivative, although affecting the mean-square displacement, does not affect this scaling law. 


\section{CMF EQUATION WITH REACTION}

We start from the CMF equation

$$
\frac{\partial P}{\partial t}=\frac{4 D_{0}}{d_{w}^{2} x^{d_{f}-1}} \frac{\partial}{\partial x}\left[\left(\frac{x}{t^{1 / d_{w}}}\right)^{d_{w}-u} x^{d_{f}-d_{w}+1} \frac{\partial P}{\partial x}\right]+r P(1-P),
$$

which has been derived very recently [9].

\section{A. Reduction to a Hamilton-Jacobi equation}

After taking into account the hyperbolic scaling and the field $G^{\varepsilon}(x, t)=-\varepsilon \ln P(x / \varepsilon, t / \varepsilon)$ one has from Eq. (27)

$$
\begin{aligned}
\frac{\partial G^{\varepsilon}}{\partial t}- & \frac{4 D_{0}}{d_{w}^{2}} \varepsilon^{\alpha+1} t^{\left(u / d_{w}\right)-1} x^{-u+1}\left(d_{f}+1-u\right) \frac{\partial G^{\varepsilon}}{\partial x} \\
& +\frac{4 D_{0}}{d_{w}^{2}} \varepsilon^{\alpha} t^{\left(u / d_{w}\right)-1} x^{-u+2}\left(\frac{\partial G^{\varepsilon}}{\partial x}\right)^{2} \\
= & \frac{4 D_{0}}{d_{w}^{2}} \varepsilon^{\alpha+1} t^{\left(u / d_{w}\right)-1} x^{-u+2} \frac{\partial^{2} G^{\varepsilon}}{\partial x^{2}}-r+r e^{-G^{\varepsilon} / \varepsilon},
\end{aligned}
$$

where

$$
\alpha=u\left(1-\frac{1}{d_{w}}\right)-1
$$

The first term in the left-hand side (lhs) of Eq. (28) and the second term in the rhs of Eq. (28) are $O\left(\varepsilon^{0}\right)$. The second term in the lhs and the first term in the rhs of Eq. (28) are $O\left(\varepsilon^{\alpha+1}\right)$ while the third term in the lhs is $O\left(\varepsilon^{\alpha}\right)$. For fractals $d_{\text {min }} \geqslant 1$, so that $u \geqslant d_{w} /\left(d_{w}-1\right)$ or $\alpha>0$. Therefore, in the limit $\varepsilon \rightarrow 0$ one has $G(x, t)=\lim _{\varepsilon \rightarrow 0} G^{\varepsilon}(x, t)$, $\lim _{\varepsilon \rightarrow 0} f\left(e^{-G^{\varepsilon} / \varepsilon}\right)=0$, provided $G^{\varepsilon}(x, t)>0$ and

$$
O\left(\varepsilon^{\alpha+1}\right) \ll O\left(\varepsilon^{\alpha}\right) \ll O\left(\varepsilon^{0}\right) .
$$

By keeping the terms up to $O\left(\varepsilon^{\alpha}\right)$, one has

$$
\frac{\partial G}{\partial t}+\frac{4 D_{0}}{d_{w}^{2}} \varepsilon^{-\left(u / d_{w}\right)+u-1} t^{\left(u / d_{w}\right)-1} x^{-u+2}\left(\frac{\partial G}{\partial x}\right)^{2}+r=0 .
$$

Equation (29) is the Hamilton-Jacobi equation for the problem (27) and may be solved by using Hamilton's equations

$$
\frac{d x(\tau)}{d \tau}=\frac{\partial H}{\partial p(\tau)}=\frac{8 D_{0}}{d_{w}^{2}} \varepsilon^{-\left(u / d_{w}\right)+u-1} \tau^{\left(u / d_{w}\right)-1} x(\tau)^{-u+2} p(\tau)
$$

$$
\begin{aligned}
\frac{d p(\tau)}{d \tau}= & -\frac{\partial H}{\partial x(\tau)} \\
= & -\frac{4 D_{0}}{d_{w}^{2}}(2-u) \varepsilon^{-\left(u / d_{w}\right)+u-1} \tau^{\left(u / d_{w}\right)-1} \\
& \times x(\tau)^{-u+1} p^{2}(\tau) .
\end{aligned}
$$

Integrating Eq. (30), one has $x(\tau)=x(\tau / t)^{2 / d_{w}}$ under the boundary conditions $x(0)=0, x(t)=x$, and

$$
L(x, \tau)=\frac{1}{4 D_{0}} \varepsilon^{\left(u / d_{w}\right)-u+1} x^{u} \tau^{\left(u / d_{w}\right)-1} t^{-\left(2 u / d_{w}\right)}-r .
$$

Finally, from Eq. (13) we obtain

$$
G(x, t)=\frac{d_{w} \varepsilon^{\left(u / d_{w}\right)-u+1} x^{u} t^{-\left(u / d_{w}\right)}}{4 D_{0} u}-r t .
$$

If one inverts the hyperbolic scaling in Eq. (31), by taking $x \rightarrow \varepsilon x$ and $t \rightarrow \varepsilon t$, one has

$$
G(x, t)=\left(\frac{d_{w} x^{u} t^{-\left(u / d_{w}\right)}}{4 D_{0} u}-r t\right) \varepsilon .
$$

The speed of the front is computed from $G(x, t)=0$, and after inverting the hyperbolic scaling one finally has

$$
\begin{aligned}
v_{H J}(t) & =\frac{d x}{d t}=\left(\frac{1}{u}+\frac{1}{d_{w}}\right)\left(\frac{u}{d_{w}}\right)^{1 / u}\left(4 r D_{0}\right)^{1 / u} t^{\left(1 / d_{w}\right)-1+1 / u} \\
& \sim t^{\left(1 / d_{\min }\right)-1} .
\end{aligned}
$$

It is important to note that the scaling law in Eq. (32) does not depend on $d_{f}$ and $d_{w}$ but only on $d_{\text {min }}$

\section{B. Local-equilibrium approach}

Following the same method as in previous sections, we find for Eq. (27)

$$
v_{L E}=\frac{4 \sqrt{D_{0} r}}{d_{w}} \frac{x^{-(u / 2)+1}}{t^{(1 / 2)-\left(u / 2 d_{w}\right)}}-\frac{4 D_{0}}{d_{w}^{2}}\left(d_{f}-u+1\right) \frac{x^{-u+1}}{t^{1-u / d_{w}}},
$$

which leads us to the front speed

$$
\begin{aligned}
v_{L E} & =\left(\frac{1}{u}+\frac{1}{d_{w}}\right)\left(\frac{4}{1+\frac{d_{w}}{u}}\right)^{2 / u}\left(r D_{0}\right)^{1 / u} t^{\left(1 / d_{w}\right)-1+1 / u} \\
& \sim t^{\left(1 / d_{\min }\right)-1} \text { for } t \rightarrow \infty
\end{aligned}
$$

It is interesting to note that although the LE prediction (34) has the same scaling law as the HJ result (32), the factor is different unless $d_{w}=2 d_{\min }$. However, we have checked numerically that for the typical ranges of $d_{w}$ (from 2 to 4 ) and $d_{\text {min }}$ (from 1 to 2 ) in fractals, the differences between the two models are negligible (see Fig. 2). 


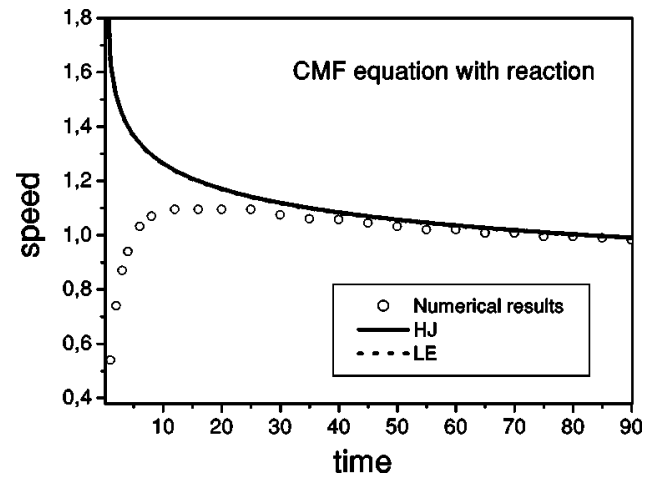

FIG. 2. Comparison between numerical results for the speed of fronts of Eq. (27) and the analytical results from the HJ method given in Eq. (32) (solid line) and the numerical result for the LE method obtained from Eq. (34) with $d_{f}=1$. All magnitudes are dimensionless. There is good agrement between analytical and numerical solutions in the asymptotic regime. We have taken $D^{*}=r$ $=1$ and $d_{w}=2.32, d_{\text {min }}=1.1$.

\section{DISCUSSION}

After all the mathematical formalism, the main conclusion we obtain is that, despite all these equations seeking to describe the features of transport on fractals, the characteristics of the fronts predicted in the four cases shown are clearly different. First of all, we should note that a diffusion equation on fractals must agree with the results (2)-(4). We have shown [9] previously that equation CMF is the only one that can exactly reproduce them, so we expect that the fronts predicted by this equation correspond to the real case.

One may wonder why fronts on fractals should be accelerated. To answer this we need to introduce in our discussion the "chemical distance" $l[11]$, which is defined as the shortest path between two points belonging to the fractal (Fig. 3).

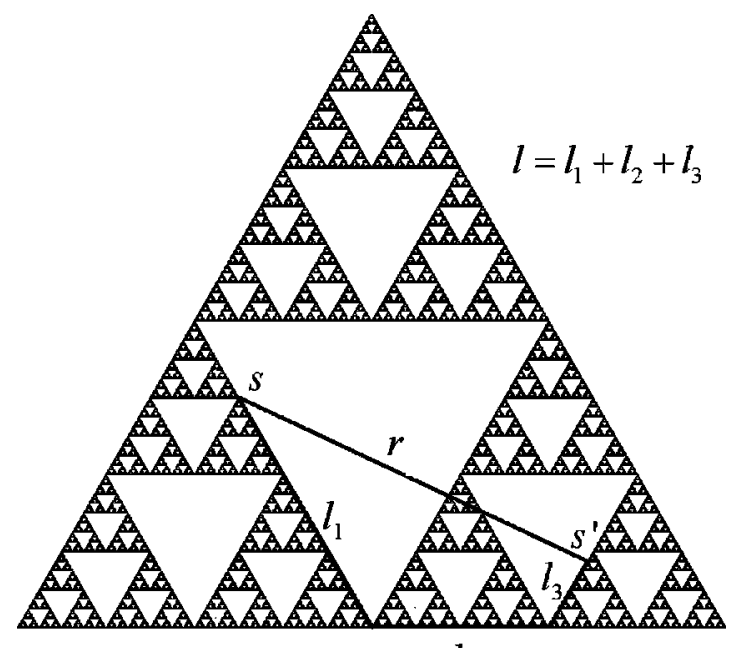

$l_{2}$

FIG. 3. Sierpinski gasket as an example of random walk on a fractal. The bold lines show the difference between the Euclidean distance $r$ and the "chemical distance" $l$ between two points of the fractal $s$ and $s^{\prime}$ (note that $l \geqslant r$ for any couple of points).
Usually, random walks (or diffusion) on a fractal is described as follows. A particle at point $s$ (see Fig. 3) can jump to every one of its first neighbors with the same probability. At the next time step, it may again jump to the new first neighbors with the same probability, and so on. We can see that the possible points reached after two jumps forward have in common that their chemical distance to the origin $s$ is the same (but not their Euclidean distance). Hence, we see that transport on fractals take place through the chemical distance space and in this space the fractal fronts are expected to show constant speed, as in homogeneous media.

Nevertheless, we are usually interested in the results for the Euclidean space, so we need the well-known relationship between $r$ and $l$ [11],

$$
l \sim r^{d_{\min }}
$$

where the condition $d_{\text {min }} \geqslant 1$ comes directly from the fact that $l$ is always greater than $r$, as seen in Fig. 3. From this expression we can conclude that, assuming that fronts advance at a constant speed in the chemical distance space, they cannot do so in the Euclidean space. Moreover, we observe that this behavior is due to the parameter $d_{\min }$ (in the nonfractal case we have $d_{\min }=1$, so the behavior in both spaces will then be the same); it agrees with the CMF equation (27), which predicts an acceleration dependence only on $d_{\text {min }}$ [see Eq. (32) or (34)], while the other equations analyzed (Secs. II, III, and IV) do not take into account this essential parameter.

In fact, the form of the time exponent in Eqs. (32) and (34) can be justified. We may define $l_{f r}$ and $r_{f r}$ as the chemical and Euclidean distances of the wave front position, respectively. As the front speed is constant in the chemical distance space, we can assume that $l_{f r}$ grows linearly with $t$. This relation, in addition to Eq. (35), leads to

$$
r_{f r} \sim t^{1 / d_{\min }}
$$

and the time dependence expected for the fronts in the Euclidean space is then

$$
v \sim \frac{r_{f r}}{t} \sim t^{\left(1 / d_{\min }\right)-1}
$$

in agreement with the acceleration predicted by the $\mathrm{CMF}$ equation [Eq. (32) or (34)]. This result, which had been already predicted for the speed of propagation of fronts in percolation clusters [18], should be valid for all those transport processes on fractals which, as it happens in most cases, take place through the chemical distance space (as in Fig. 3).

In conclusion, we have shown that the CMF equation not only describes the features of diffusion on fractals better than previous ones [9], but it also predicts some essential features of the propagative processes on those heterogeneous media, i.e., the speed and acceleration of the wave fronts derived when a reaction (logistic) process, widely used in biophysics [19], is considered. In consequence, we think that CMF equation is the best analytical approach proposed to date for description of transport on fractals. 
One of the main results presented here is the wave front speed (32), which shows that the acceleration is determined by the parameter $d_{\text {min }}$, as argued theoretically above. It is interesting to note that this parameter is not the same as that responsible for anomalous diffusion, namely, $d_{w}$ [see Eq. (2)], contrary to what one could expect. We think that an exhaustive analysis of the meaning of the parameter $d_{w}$,

[1] D. Campos, J. Fort, and J.E. Llebot, Phys. Rev. E 66, 062901 (2002).

[2] J. Fort and V. Méndez, Phys. Rev. Lett. 82, 867 (1999).

[3] V. Méndez and J.E. Llebot, Phys. Rev. E 56, 6557 (1997).

[4] N. Shigesada and K. Kawasaki, Biological Invasions: Theory and Practice (Oxford University Press, New York, 1997).

[5] P.K. Burgess et al., J. Neuropathol. Exp. Neurol. 56, 704 (1995).

[6] A.M. Kilpatrick and A.R. Ives, Nature (London) 422, 65 (2003); D.L. Smith et al., Proc. Natl. Acad. Sci. U.S.A. 99, 3668 (2002).

[7] O.N. Bjornstad et al., Science 298, 1020 (2002).

[8] S. Alexander and R. Orbach, J. Phys. (Paris), Lett. 43, 2625 (1982).

[9] D. Campos, V. Méndez, and J. Fort (unpublished).

[10] D. Stauffer and A. Aharony, Introduction to Percolation Theory, 2nd ed. (Taylor \& Francis, London, 1994).

[11] A. Bunde and S. Havlin, Fractals and Disordered Systems which has been traditionally defined just from Eq. (2), is still needed. This and many other questions which have not been explained theoretically yet [18] show very clearly that there is still a lot of work to do on fractal dynamics. Although theoretical research on fractals decreased after a boom in the early 1980s, we consider that efforts in this field, as the one presented here, are still strongly useful.

(Springer-Verlag, Berlin, 1991).

[12] B. O’Shaughnessy and I. Procaccia, Phys. Rev. Lett. 54, 455 (1985).

[13] M. Giona and H.E. Roman, Physica A 185, 87 (1992); J. Phys. A 25, 2093 (1992); H.E. Roman, Phys. Rev. E 51, 5422 (1995).

[14] R. Metzler, W.G. Glöckle, and T. Nonnenmacher, Physica A 211, 13 (1994).

[15] V. Méndez, J. Fort, H. G. Rotstein, and S. Fedotov, Phys. Rev. E 68, 041105 (2003).

[16] S. Fedotov and V. Méndez, Phys. Rev. E 66, 030102(R) (2002).

[17] V. Méndez, J. Fort, and J. Farjas, Phys. Rev. E 60, 5231 (1999).

[18] D. ben-Avraham and S. Havlin, Diffusion and Reactions in Fractals and Disordered Systems (Cambridge University Press, Cambridge, 2000).

[19] J. Fort and V. Méndez, Rep. Prog. Phys. 65, 895 (2002). 